Int. J. Speleol. 10 (1978), pp. 167 - 178

\title{
Neue Ergebnisse speläologischer Untersuchungen in den Hainburger Bergen (NÖ) und ihre wissenschaftliche Bedeutung
}

\author{
von
}

KARL MAIS*

\begin{abstract}
New results of the speleological researches in the HAINBURGER BERGE (Lower Austria) and
\end{abstract} their scientific meaning.

\section{SUMMARY}

Since 1971 speleological and paleontological investigations have been carried out in the quarry «Hollitzer» wich is in the Pfaffenberg near Bad Deutsch Altenburg (Hainburger Berge, Lower Austria). Evident karst phenomena are missing at the surface, but the existence of caves filled with sediments in the quarry show that the area is a fossil buried karst.

A steady scientific survey allowed the study of the karstic phenomena without hindering the work in the quarry. Up to now more than 150 cavities have been discovered. Most of them seem to belong to a unique solutional system; corrasive forms are missing.

Fossils (Gasteropoda, Amphibia, reptiles, birds and mammals) have been found in more than twenty cave fills. The oldest findings can be dated from the middle to the recent Pliocene (Csarnotanum), the more recent ones to the older Pleistocene (Villanyium; Biharium) the material is well preserved. Its variety in species and individuals as well as the possibility for a biostratigraphical evaluation makes the findings of Pfaffenberg a particular source for the above mentioned periods. The investigations are still continuing.

\section{EINFÜHRUNG UND ÜBERBLICK}

Im Osten Österreichs, rund $40 \mathrm{~km}$ östlich der Bundeshauptstadt Wien und rund $15 \mathrm{~km}$ westlich der slowakischen Hauptstadt Bratislava, liegen die Hainburger Berge (Abb.l).Zwischen ihnen und den nördlich anschliessenden Kleinen Karpaten verlässt die Donau unterhalb der Marchmündung, durch die Engtalstrecke der Hainburger Pforte das Wiener Becken, um in das Pannonische Becken einzutreten.

Die Hainburger Berge erheben sich als Inselgebirge bis rund 200 Meter über die Ebene des östlichen Wiener Beckens. Zu ihnen gehören der Hundsheimer Berg (480 m Seehöhe), der westlich etwas abgesetzte Pfaffenberg (340 m See-

\footnotetext{
* Abteilung für Höhlenschutz am Bundesdenkmalamt, A-1010 Wien, Hofburg, Säulenstiege (Austria)
} 


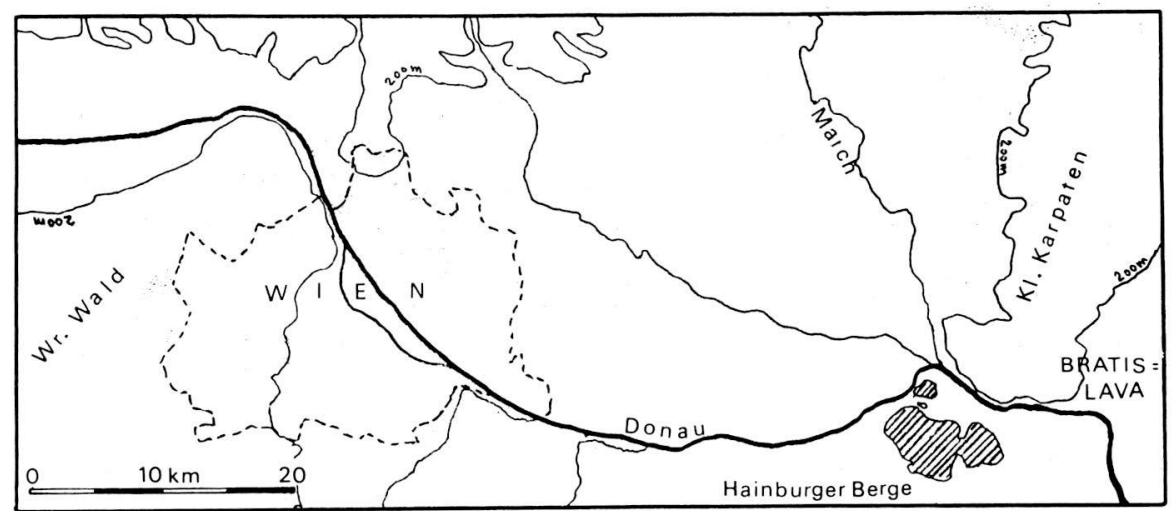

Abb. 1.: Lage der Hainburger Berge.

höhe), der Hainburger Schlossberg, ein ehemaliger Umlaufberg und der nördlich vorgeschobene, ebenfalls stärker isolierte Braunsberg.

Auf einem Sockel aus kristallinen Gesteinen - Granite, Schiefer und Quarzit liegen fossilleere, dolomitische Kalke mesozoischen Alters. Randlich sitzen Strandkonglomerate und Leithakalke auf.

Die Berge erheben sich, besonders in den westlichen Teilen, mit einer steilen Hangpartie aus der Erbene und führen dann flacher werdend zu den Kuppen, die die höchsten Erhebungen darstellen. Im Osten steht dichter Wald auf tiefem Boden. Gegen Westen, besonders an Steilhängen, tritt der felsige Untergrund zutage. Er ist bisweilen nur von einer dünnen Bodenschichte bedeckt. An geeigneten Stellen ist Hanglöss aufgeschlossen.

Das Gebiet der Hainburger Berge war bereits früher durch einige Höhlen und das Fehlen einer oberirdischen Entwässerung als Karstgebiet gekennzeichnet. Aufallend ist, dass oberirdische Karstformen im Landschaftsbild kaum in Erscheinung treten. Die Hänge sind im allgemeinen nur durch flache, an den Steilhängen tiefere Erosionsrinnen gegliedert.

Das Ausmass der tatsächlichen Verkarstung konnte erst in den letzten Jahren, im Zuge intensiver Geländearbeiten erkannt werden.

In ausgehenden 19. Jahrhundert lieferten mehrere kleine Steibrüche am westlichen Rand der Hainburger Berge, im Bereich der Gemeinden Bad Deutsch-Altenburg und Hundsheim, Kalkegestein für kleine Kalköfen und die Steingewinnung. In diesen Brüchen kam es dabei wiederholt zur Aufschliessung und Entdeckung von Höhlen, deren grösste und bedeutendste die Güntherhöhle (Kat. Nr. 2921/2, ist. Sie besitzt eine Länge von rund 80 Metern und steht in direktem Zusammenhang - durch eine sinterbedeckte Höhlenwand -mit der Knochenspalte von Hundsheim (Kat. Nr. 2921/13). Diese ist im Altquartär durch einen Deckensturz schachtartig geöffnet worden und hat als Tierfalle gewirkt. Abgestürzte Tiere und eingebrachte Sedimente haben diesen 
Höhlenteil vollständig erfüllt.

Bei mehreren Grabungen, die ab der Jahrhundertwende eingesetzt haben, ist immer wieder ergiebiges paläontologisches Fundgut zutage gefördert worden. Über diese Funde gibt es eine Reihe von Veröffentlichungen, auf die bei Rabeder (1972c) hingewiesen ist.

Während an den kleinen Steinbrüchen im Bereich der Gemeinde Hundsheim der Betrieb mit der Zeit eingestellt worden ist, entwickelte sich der Steinbruch der «Hollitzer Baustoffwerke» in Bad Deutsch-Altenburg wegen seiner günstigen Lage an der ehemals sehr bedeutenden Eisenhahnlinie Wien-Bratislava (Press-burgerbahn) stetig weiter. Heute ist er der grösste und leistungsfähigste Betrieb für Bruchstein und Schotter in dieser Region.

Wiederholt kam es in diesem Steinbruch am Pfaffenberg zur Aufsprengung von karst-und höhlenkundlichen Objekten, die bedauerlicherweise nie speläologisch untersucht wurden. Einem glücklichen Zufall ist es zu verdanken, dass im Jahre 1971 bemerkenswerte Karstformen und reiche Knochenfunde bekannt und einer grüdlichen wissenschaftlichen Untersuchung zugänglich geworden sind (Mais 1972). Hatte es anfangs den Anschein, als wäre mit einer blossen Bergung von Fossilien und einer Dokumantation der spelälogischen Objekte das Auslangen zu finden, so zeigte sich bald, dass eine kontinuierliche Beobachtung des Steinbruches erforderlich wäre und ausserordentliche Erfolge liefern könnte.

Dank der forschungsfreundlichen Einstellung von Eigentümern und Betriebsleitung der Hollitzer Baustoffwerke konnten die Höhlen und die Sedimente untersucht und beprobt werden. An diesen Arbeiten waren Mitarbeiter des Naturhistorischen Museums Wien, des Landesvereins für Hölenkunde in Wien und Niederösterreich, der Krahuletz Geselschaft Eggenburg und im Wesentlichen des Paläontologischen Institus der Universität Wien und der Höhlen-abteilung des Bundesdenkmalamtes beteiligt.

Die Geländearbeiten lieferten grosse Mengen fossilführender Sedimente, die oft eine sehr langwierige und aufwendige Ausarbeitung, durch eine chemische Aufschließung, durch Schlämmen der gelockerten Sedimente und Aussuchen der Fossilien aus dem Schlämmrückstand erforderlich machten. Diese grossen personellen und finanziellen Belastungen, die vom Paläontologischen Institut getragen wurden, liessen den Wunsch aller an den Forschungen Beteiligten nach einer gezielten Förderung wach werden.

Es wurde ein Forchungsprogramm ausgearbeitet und zu Begim des Jahres 1974 von Hofrat Prof. Dr. Friedrich Bachmayer, Dr. Gernot Rabeder und dem Berichterstatter beim «Fonds zur Förderung der wissenschaftlichen Forschung in Österreich» eingereicht. Diesem Vorhaben wurden für drej Jahre die erforderlichen Mittel zugestanden. Wegen der Entdeckung immer wieder neuer und ergiebiger Fossilfundstellen und Höhlenobjekte reichten Dr. Gernot Rabeder und der Berichterstatter ein Nachfolgeprojekt ein, das nunmehr 


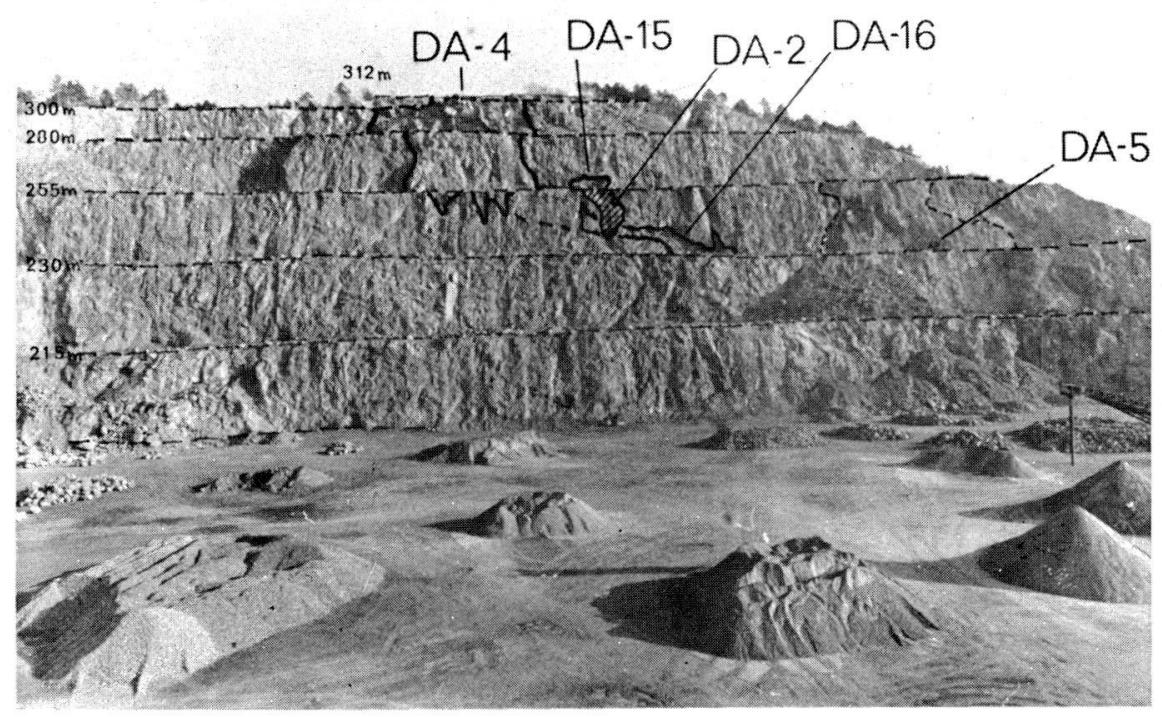

Abb. 2.: Steinbruch «Hollitzer» im Pfaffenberg bei Bad Deutsch-Altenburg, Westteil der Hainburger Berge, NÖ.- Schachtzone (Bildmitte) mit Objekten DA-4,-15,-2 und -16, sowie Höhlenbereich um DA-5 (rechts). Objekte der Schachtzone durch schrägen Blickwinkel nach links verschoben. Situation vom November 1977.

die weiteren Forschungen bis zum Jahre 1979 sicherstellt.

\section{ALLGEMEINE BEOBACHTUNGEN IN DEN HÖHLEN}

Der Pfaffenberg ist durch den Steinbruch der Hollitzer Baustoffwerke in einer Breite von mehr als 500 Meter; und einer Höhe von rund 110 Meter aufgeschlossen (Abb. 2). Fünf horizontale Etagen machen die sechs Abbauwände gut zugänglich. In den bis zu 30 Meter hohen Abbauwänden waren zu Beginn der spelälogischen Untersuchungen fünf grosse Höhlenräume und zahlreiche kleine Objekte festzustellen. Zwei der grossen Räume, waren vollständig von Sedimenten erfüllt, die anderen zur teilweise. Die Grösse und die horizontale Verteilung der aufgeschlossenen Räume, sowie die Anzahl kleiner Objekte hat bereits damals auf eine intensive Durchhöhlung des gesamten Berges hingewiesen, in einer Art, die vorher nicht anzunehmen war.

Nach der ersten umfassenden Vermessung und Dokumentation der Höhlen und Höhlenreste im Steinbruch (Mais 1973) wurden die folgenden Aufnahmen mit dem Fortschreiten des Gesteinsabbaues weitergeführt. Es war dabei zu erkennen, dass verschiedene Objekte in Verbindung zueinander standen und dass durch neue Sprengungen manche Höhlen immer tiefer in den Berg zu verfolgen waren. 
Bei Erdarbeiten auf dem Rücken des Pfaffenberges, die zur Freilegung der Gesteinsoberfläche als Vorbereitung für das Zurückversetzen der obersten Steinbruchsetage dienten, ist mi Jahre 1971 eine vorher nicht erkennbare «dolinenartige» Bildung aufgedeckt worden. Diese war beim Nachrücken der Abbaukante als schachtartige Hölenfüllung zu erkennen. Beim weiteren Abbau hat sich nach Jahren gezeigt, dass dieses Objekt vom Rücken des Pfaffenberges in $312 \mathrm{~m}$ Seehöhe, als gewaltiger Schacht in die Tiefe zieht, zur Zeit bis in eine Tiefe von - 80 Meter. Grosse Versturzblöcke füllen sein Lumen; fluviatile Sande sind von oben her eingebracht und füllen die Zwischenräume weitgehend aus. Bisweilen stehen grössere Lückenräume unterhalb oder zwischen den Blöcken frei. Diese Räume sind manchmal mehere Meter weit zu befahren und zeigen am Sediment ihrer Sohle zahlreiche Fledermausknochen. Daraus ergibt sich, dass diese Spalten Fledermäusen als Hangplatz gedient haben und dass die Sedimente nicht in den Schacht «eingeschlemmt» worden sind.

Im obersten Teil des Schachtes war Löss als Füllung anzusprechen. Er war von einer grob kantengerundeten Stein- und Erdlage, der letzten Sedimentschichte unter dem rezenten Humushorizont, überdeckte. Es hat den Anschein, als hätte eine Solifluktionsmasse zur vollkommenen Plombierung des Schachtes geführt.

Seitenteile des Schachtes stehen mit anderen, früher als eigene Höhlen aufgefassten Objekten in Verbindung. Diese sind stets im schachtwärtigen Teil von Sedimenten erfüllt, während sie in ihren peripheren Teilen oft eine sehr geräumige Konvakuation beseitzen.

Dem Schacht, als Fossilfundstelle «DA-4» benannt, gehört derzeit auch die Fundstelle DA-2 und die mit dieser direkt zusammenhängenden Fundestelle DA-16, einer Tropfsteinhöhle, an. Neben dem räumlichen Zusammenhang sind ihre Sedimente auch stratigraphisch trennbar, bezw. abfolgend (Rabeder 1978).

DA-16 steht mit der Fundstelle DA-15 in räumlichem Zusammenhang. Beides sind/waren relativ grossräumig begehbare Höhlen aus denen der Raumcharakter zu erkennen ist, siehe Abb. 3.

Die Höhlenstrecken zeigen eine deutliche Bindung an SE/SSE streichende Klüfte, bezw. an eine annährend normal hiezu laufende Klüftung. Kleinere Partien haben enge, sehr hohe Profile, an deren Verengungen durch Klemmblöcke Zwischenböden entstanden sind. Am Zusammentreffen derartiger Partien sind mehrfach grössere Räume entwickelt. Grosse Räume, wie bei DA-15 lassen keine Zusammenhänge mit gangförmigen Höhlenteilen erkennen. Im nordwestlichen Vorfeld von DA-15 waren früher mehrfach große Räume gelegen, die zur Fundstelle DA-2 gehört haben und weitgehend sedimenterfüllt waren. Auch sie haben den Eindruck erweckt, mehr oder minder abgeschlossene Hallen zu sein und nicht Teile eines Gangsytems.

Etwas südlicher der oben erwähnten Höhlenräume liegt das Gebiet der Fund- 


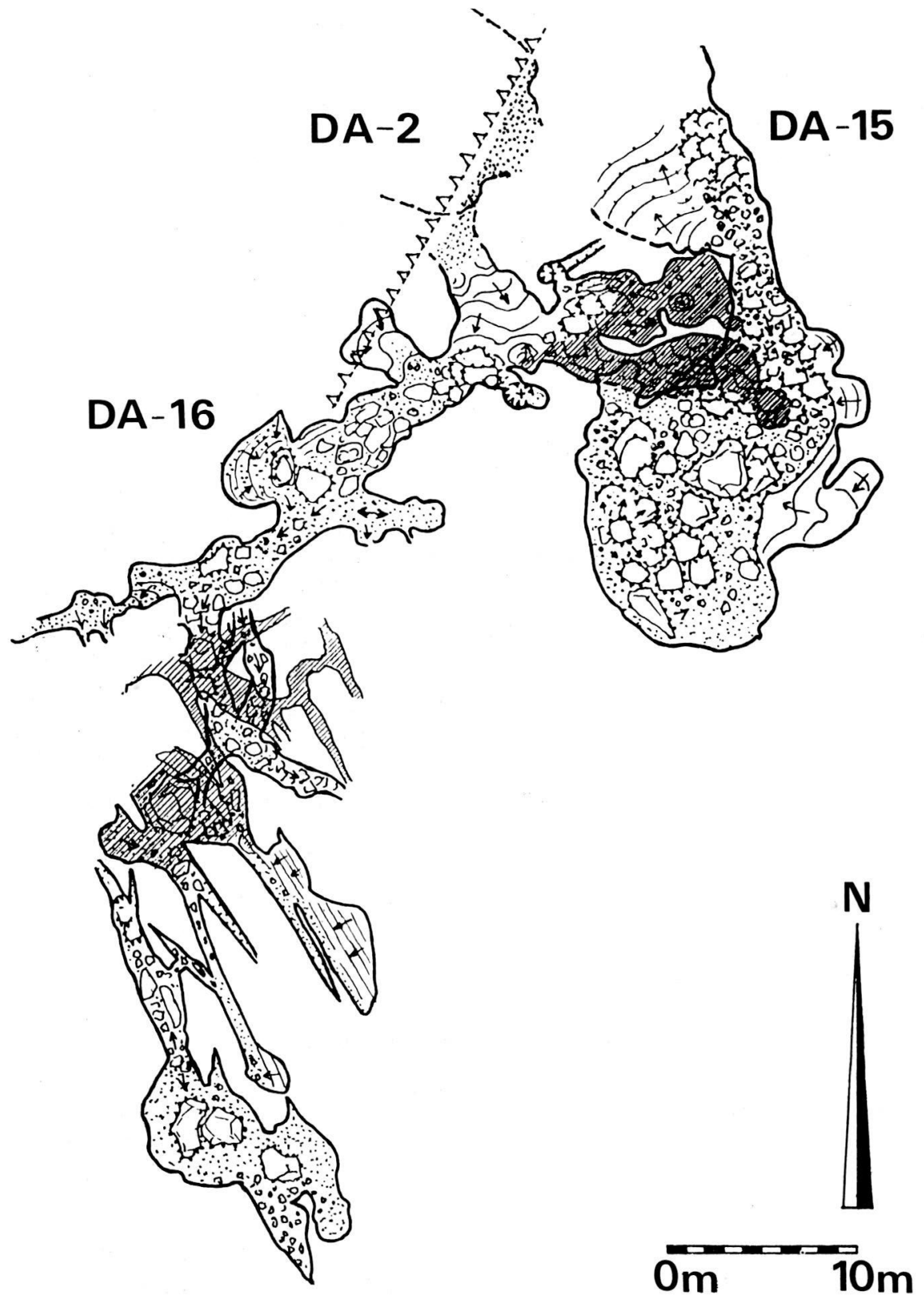

Abb. 3.: Schachtzone, mit Räumen der Objekte DA-2,-15 und -16. Die Fundstelle DA-2 ist an der Abbaukante (strichlierte Pfeillinie) aufgeschlossen. Ihre Sedimente stehen mit DA-16 in Verbindung. Der norwestlich verlaufende Teil von DA-16 weist reiche Tropfsteinbildungen auf und besitzt eine Verbindung zum grossen Raum von DA-15.

Die Raumfolge ist aus Teilvermessungen verschiedener Abbauphasen zusammengezeichnet, vermessene Länge $275 \mathrm{~m}$. Unterlagerende Hölenräume schraffiert dargestellt. 


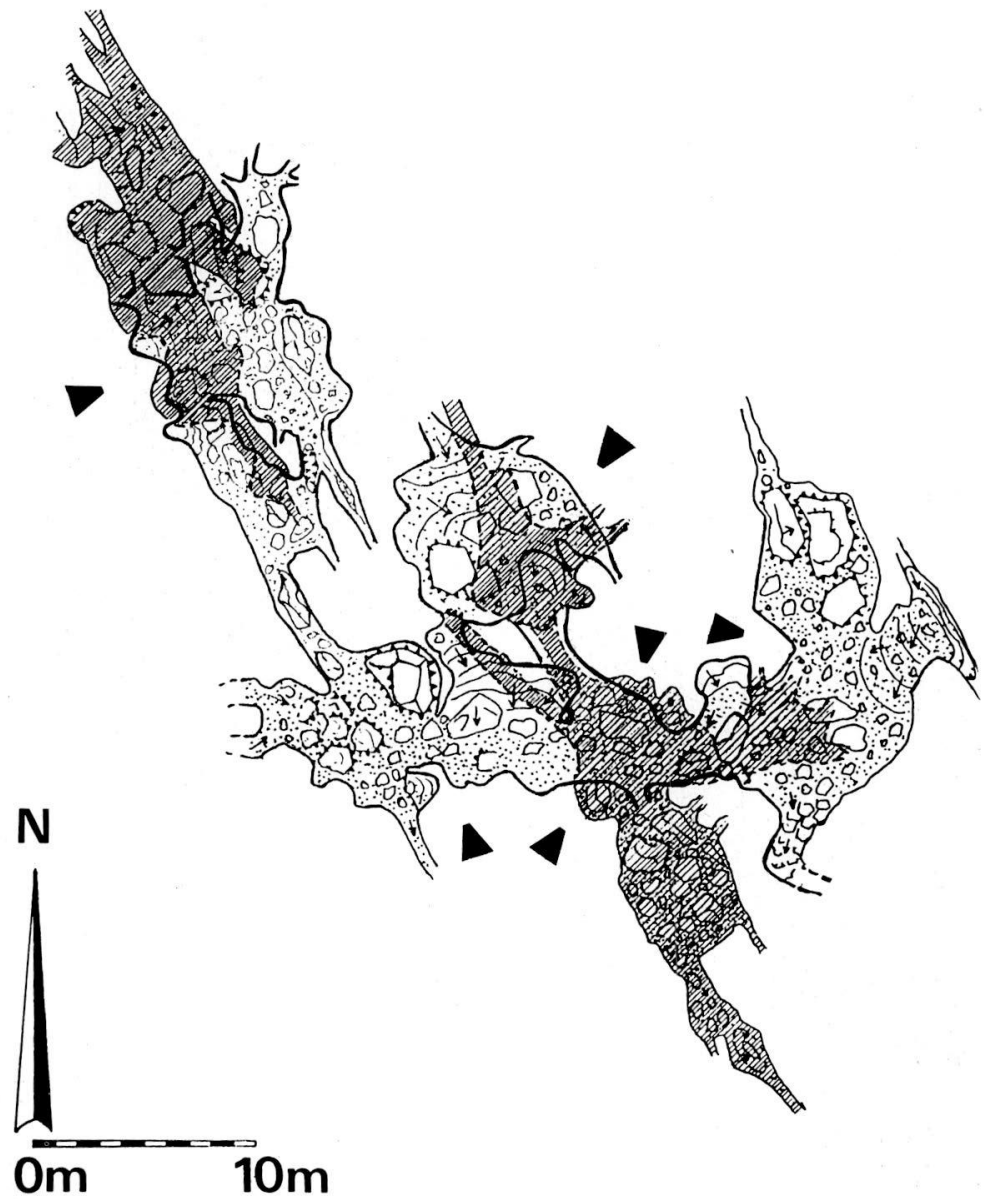

Abb.4.: Höhlenräume im Bereich der Fundstelle DA-5. Die Anlage der Räume an SE/SSE streichende Klüfte deutlich sichtbar.

Auffallend die Anzahl von 11 (elf) hallenartigen Räumen mit Raumhöhen zwischen 3 und 6 Metern, die sehr nahe nebeneinander und übereinander stehen (Pfeile).

Die dargestellte Höhlenpartie ist rund $250 \mathrm{~m}$ lang und war durchgehend befahrbar. Unterlagende Höhlenräume schraffiert dargestellt.

stellen DA-5, DA-6, und DA-19, siehe Abb. 2, die sehr nahe beinander liegen (lagen) und in räumlichem Zusammenhang zu sehen sind. Die Höhlenteile dieses Bereiches weisen ebenso eine Bindung an die SSE Klüftung auf, wie die nördlich gelegene Schachtzone; die Räume sind hier jedoch weit größer entwickelt. Auffälling ist das sehr nahe Zusammenstehen der Hallen : zum Teil nebeneinander, zum Teil übereinander, sie sind einander dabei so nahe, dass sie durch Klufterweiterungen und Kolkbildungen gute Verbindungen unter- 
einander aufweisen, siehe Abb. 4.

Die vertikale verteilung der Höhlen ist im Steinbruch nicht auffällig. Verfüllte oder begehbare Räume sind in allen Höhen vorhanden. Horizontal fällt demgegenüber auf, dass im Norden eine eher höhlenarme Zone mit nur gelegentlich, grossen Räumen liegt, während im Süden zahlreiche Objekte unterschiedlicher Ausdehnung vorhanden sind. Es wäre möglich, dass die südlichen Höhlenräume zu einem einzigen Höhlensystem gehören, dessen Verbindungen jedoch wegen der methodischen Schwierigkeiten der Erfassung nur unvollständig bekannt geworden sind.

In diesem Zusammenhang ist hervorzuheben, dass die Befahrbarkeit der meisten Höhlenräume erst durch mühevolles Ausräumen von Sprengschutt aus «höhlenverdächtigen» Stellen erzwungen werden musste. Mehrfach war auch festzustellen, daß grössere Hölen nach Folgesprengungen nicht mehr zugänglich waren, sie waren offensichtlich durch die Sprengwirkung zusammengestürzt.

Die dokumentierten Höhlen stellen somit einen «Mindestbestand» an Höhlen dar.

Eine Gesamtdarstellung der bekannt gewordenen Höhlenobjekte wird vorbereitet. Dann kann die Verteilung der Objekte im untersuchten Areal ersehen und besser beurteilt werden.

Für die Entstehung der Höhlen am Pfaffenberg waren bisher keine Anzeichen einer erosiven Bildungsphase zuerkennen. Erosionsprofile, Fliessfazetten oder gangartige Höhlenräume fehlen. Die Korrison scheit die ausschlaggeben de Kraft der Raumbildung gewesen zu sein. Die Höhlenräume sind meist klar an die Klüftung gebunden, Raumerweiterungen verbinden vielfach parallelziehende Klufträume miteinender. Domartige Kolkräume sind einander eng benachbart und oft auch verbunden. Die Bildung dieser Höhlen im Pfaffenberg dürfte am ehesten im phreatischen Bereich erofolgt sein. Die weitere Entwicklung dürfte danach in eine oder Phasen einer vollständigen Erfüllung mit Wasser bei schwacher Wasserbewegung zu stellen sein.

Die Höhlen zeigen oft Reste einer Tropfsteinbildung. Es sind Decken-, Wand -und Bodenformen anzutreffen. Ein besonderes Gepräge besitzen viele Höhlenwände im Besatz weiter Flächen mit Knötchensinter; manche Klüfte sind dadurch kaum befahrbar.

Die Wandversinterungen sind grossteils als präquartat einzustufen, da sie ober und unterhalb gut datierbarer Sedimenfüllungen in vergleichbarer Weise entwickelt sind. An manchen Stellen sind jüngere Bildungen vorhanden, rezente Tropfsteine scheinen zu fehlen.

\section{SPEZIELLE UNTERSUCHUNGEN IN DEN HÖHLEN}

Die Erfassung und Dokumentation der Höhlen stellt jedoch nicht die Haup- 


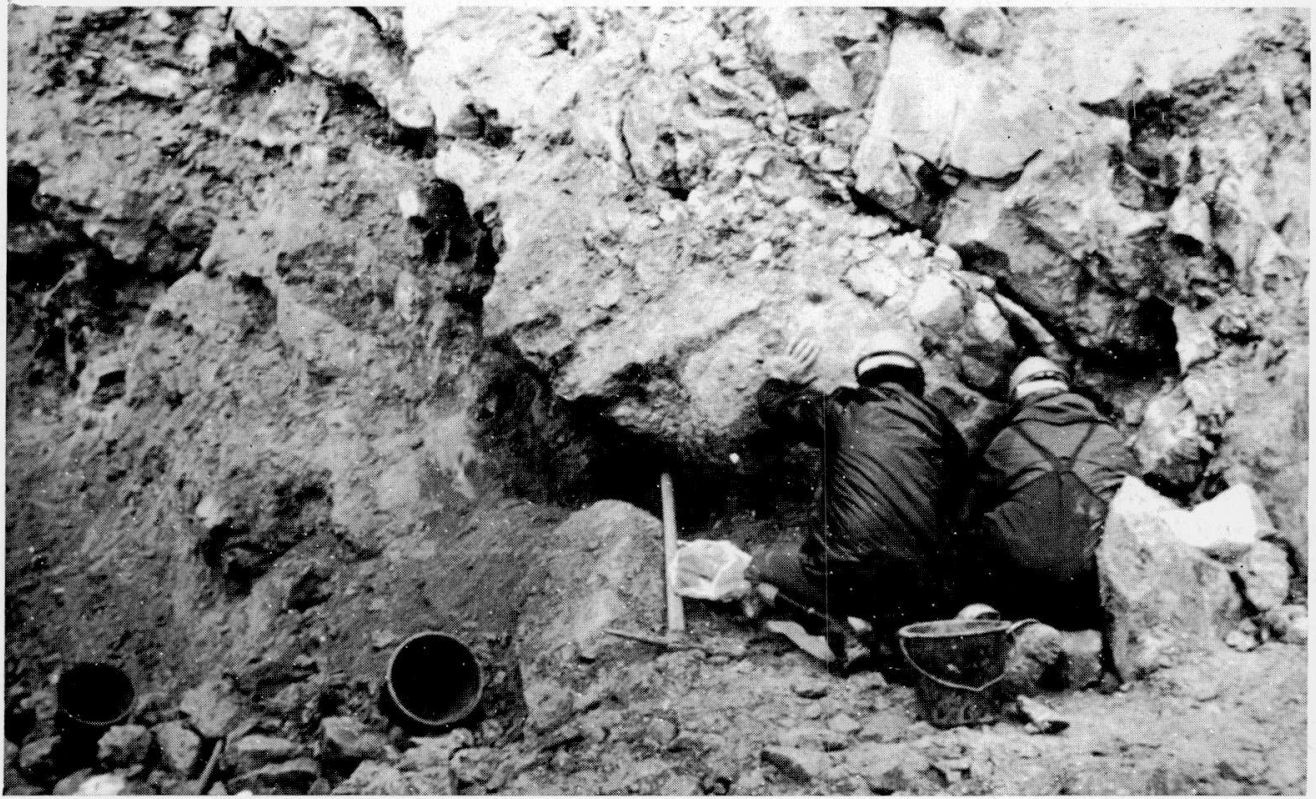

Abb. 5.: Fundstelle DA-4, oberer Teil des Schachtes, 300m Seehöhe. Sedimentfüllung mit grossem Versturzblock, reiche Fossilfundstelle, unter anderem mit Bär, Bison und Pferd. Situation vom 17.10.1975.

taufgabe dar. Es wurde daher von der Projektleitung immer wieder versucht verschiedene fachspezifische Untersuchungen anzuregen.

Sedimentpetrographische Analysen der oberen Partie des Schachtes liegen von der Gerhard Niedermayer und Robert Seemann vor (1974).

Biospeläologische Bemühungen des Berichterstatters waren bisher weder an Köderfallen, an ausgelegten Ködern noch an Wasserstellen erfolgreich. Ebenso konnten keine Hinweise auf rezente Besuche von Fledermäusen gefunden werden. Diese ungewöhnlichen, negativen Befunde sprechen für eine vollständige Plombierung des Pfaffenberges mit sehr feinen Sedimenten, durch die den subterranen Lebensgemeinschaften das Eindringen in die Höhlen unmöglich gemacht und sogar die Nahrstoffzufuhr von außen abgeschnitten wurde. Pollenanalythische Untersuchungen von Dr. Ilse Draxler waren bisher ebenso negativ. Dies ist jedoch auf das locker sandige Probenmaterial zurückzuführen, in dem eine Erhaltung von Pollenkörnern recht unwahrscheinlich war. Es wird nach günstigerem Material gesucht.

Die reichsten und bedeutendsten Ergebnisse lieferten bisher die paläontologischen Forschungen. Sie lagen stets in den Händen von Univ. Doz. Dr. Gernot Rabeder.

Bis Ende 1977 konnten in 20 Höhlenfüllungen Fossilien entdeckt werden, den Fundstellen DA-2 bis DA-21. Die Fundstelle DA-1 wurde um die Jahrhundertwende aufgefunden und von Wilhelm Freudenberg 1914 (Geol.u.Paläont. Abh. (Jena) NF 12 (4/5)) bearbeitet; 1971 bestand sie nicht mehr. 
Bei den informativen Begehungen des Steinbruches wurden stets die Sedimentfüllungen auf ihren Fossilbestand untersucht. Wenn Knochenreste zu erkennen waren, erfolgten Probenentnahmen und Schlämmanalysen im Labor, bei positivem Ergebnis eine Grabung. Dabei konnte wegen des überwiegenden Anteils von Mikrovertebraten am Fundgut und des guten Erhaltungszustandes das Sediment zügig abgegraben und in Säcken verstaut werden. Nach entsprechender Vorbehandlung folgte das Schlämmen im Labor. Das dadurch erzielte Konzentrat aus Knochen, Schneckenschalen und gröberen Sedimentelernenten wurde händisch ausgelesen.

Bei gelegentlichen Großäugerfunden wurde konventionell gegraben.

Auf diese Art und unter dem Druck immer neu entdecker Fundstellen mußte außerorderntlich viel Material geborgen und aufgearbeitet werden. Dadurch gelang es einen sehr umfangreichen Fossilbestand zu sichern, in dem zahlreiche neue Arten und Gattungen nachzuweisen waren. Dies führte zu einer Bereicherung des Faunenbestandes für Österreich und darüberhinaus für Europa.

Vom Pfaffenberg liegt Material vor, das sogar seltene Arten gut bis reichlich belegt.

Im Fossilmaterial sind Landschnecken - bisher 39 Arten (Binder 1976, 1977) -, Eidechsen, Schildkröten, Schlangen, Vögel und besonders Säugetiere - bisher rund 90 Arten - vertreten.

Von biostratigraphischer Bedeutung ist das unterschiedliche Alter der Fundstellen. Als älteste Höhlenfüllungen sind die Fundstellen DA-9 und DA-20 einzustufen, wegen der gut belegten Leitart Mimoys septimanus in das Mittelpliozän (Csarnotanum). An diesen Fundstellen dominierten Reste von Reptilien, wie Wirbel von Ophisaurus dem Scheltopusik, und verschiedenen Colubriden (Nattern).

Die meisten und ergiebigsten Fundstellen sind dem älteren Pleistozän zuzuordnen. Dabei hat sich durch die kontinuierliche Beobachtung und Vermessung der einzelnen Objekte sowie die sorgfältige Beprobung der Sedimente ergeben, dass die Fundstellen DA-16, DA-2 und DA-4 ein durchgehendes Profil von rund $80 \mathrm{~m}$ Höhe darstellen. Eine stratigraphische Bearbeitung durch Dr. Rabeder ist im Druck (1978).

In diesem Profil sind bei DA-2 Insektenfresser Nager, Hasen, Fledermäuse und Raubtiere - 14 Arten (Rabeder 1976) - gut vertreten, weiters kommen auch Naßhorn, Hirsch und Pferd vor. Bei DA-4 sind zusätzlich auch noch Bär, Bison und Elephant in der Faunenliste enthalten.

Im Zuge der taxonomischen Arbeiten von Binder $(1976,1977)$ und Rabeder (1972 b, 1973 b, 1976) wurden folgende Arten neu beschrieben

Gastropoda: Archaegopis acutus Binder 1978, von DA-4 B Klikia altenburgensis Binder 1978, von DA-4 B 


\author{
Mammalia: Dimylosorex tholodus Rabeder 1972 (b) \\ Oxyvormela maisi Rabeder 1973 (b), von DA-2 D \\ Psalidogale altenburgensis Rabeder 1976, von DA-2 C1 \\ Meles hollitzeri Rabeder 1976, von DA-2 C1
}

\begin{abstract}
Andere Neubeschreibungen und Bearbeitungen der Bestände in taxonomischer, ökologischer und stratigraphischer Hinsicht sind bereits weit fortschritten, ihre Veröffentlichung steht zum Teil bereits bevor.

Über die bisherigen Arbeiten gibt das Liberarturverzeichnis Auskunft, dort sind ausschließlich Arbeiten über die derzeitigen Forschungen am Pfaffenberg euthalten, mit Ausnahme von Rabeder 1972 c, mit Hinweisen auf die Literatur der Knochenspalte bei Hundsheim.
\end{abstract}

\title{
ZUSAMMENFASSUNG
}

Im Gross-Steinbruch der «Hollitzer Baustoffwerke» am Pfaffenberg bei Bad Deutsch-Altenburg, in den Hainburger Bergen (Niederösterreich) werden seit dem Jahre 1971 speläologische und paläontologische Untersuchungen durchgefürt. Die Oberfläche der Hainburger Berge weist keinen typischen Karstformenschatz auf. Die im Steinbruch aufgeschlossenen weitgehend mit Sedimenten verfüllten Hohlräume weisen das Gebiet als fossilen, plombierten Karst aus. Die ständige wissenschaftliche Kontrolle der Abbauwände des Steibruches hat es ermöglicht, einen Einblick in die sehr intensive Durchhöhlung zu gewinnen. Der Steinbruchsbetrieb wurde dabei nicht behindert; jeder neue und weitere Aufschluss wird laufend untersucht. Dabei sind bisher mehr als $150 \mathrm{Hol}-$ häume bekannt geworden. Die meisten scheinen einem einheitlichen Höhlensystem anzugehören, das weitgehend bis ausschliesslich korroviv entstanden ist. Eindeutige Erosionsformen fehlen.

In zwanzig Höhlenfüllungen sind bisher Fossilien gefunden worden: Reste von Gastropoden, Amphibien, Reptilien, Völgel und Säugetieren. Die ältesten Funde sind in das Mittel- bis Jungpliozän (Csarnotanum), die jüngeren in das ältere Pleistozän (Villanyium, Biharium) zu stellen. Das Knochenmaterial ist sehr gut erhalten. Sein Arten - und Individuenreichtum sowie seine biostratigraphische Auswertbarkeit macht den Pfaffenberg mit seinen Höhlen zu einem der bedeutendsten Fundorte für die belegten Zeitabschnitte.

Die Forschungen werden fortgesetzt.

\section{LITERATUR}

BINDER H., 1976 - Die pleistozäne Molluskenfauna aus Niederösterreich.- Diss. Univ. Wien, phil. Fak.

BINDER H., 1977 - Bemerkenswerte Molluskenfaunen aus dem Pliozän und Pleistozän von Niederöserreich. Beitr. z. Paläont. v. Österreich (Wien) 3:1-78.

MAIS K., 1973 - Entdeckung einer knochenspalte im Pfaffenberg bei Bad Deutsch-Altenburga (N.O.).- Höhlenkundl. Mitt. (Wien) 28:43-44

MAIS K., 1972 - Das Karstgebiet Pfaffenberg bei Bad Deutsch-Altenburg (Niederösterreich) -ein vorläufiger Überblick. Die Höhle (Wien) 24:1-8.

MAIS K. \& RABEDER G., 1977a - Eine pliozäne Höhlenfüllung im Pfaffenberg bei Bad DeutshAltenburg (Niederösterreich). Die Höhle (Wien) 28 (1):1-7.

MAIS K. \& RABEDER G., 1977b - Eine weitere pliozäne Höhlenfauna aus dem Steinbruch Hollitzer bei Bad Deutsch-Altenburg (Niederösterreich). Die Höhle (Wien) 28 (3):84-86.

NIEDERMAYR G. \& SEEMANN R., 1974 - Vorläufiger Bericht über sedimentpetrographische und mineralogische Untersuchungen an Höhlensedimenten des Karstgebietes Pfaffenberg bei Bad Deutsch-Altenburg. (NÖ). Die Höhle (Wien) 25(1):3-11. 
RABEDER G., 1972a - Eine fossile Höhlenfauna aus dem Steinbruch Hollitzer bei Bad DeutschAltenburg (Niederösterreich). Die Höhle (Wien)23:89-95.

RABEDER G., 1972b - Ein neuer Sorcide (Insectivora) aus dem Alt-Pleistozän von DeutschAltenburg 2 (Niederösterreich). N. Jb. Geol. Paläont. Mh. (Stuttgart) 1972:635-642.

RABEDER G., 1972c - Die Insectivoren und Chiropteren (Mammalia) aus dem Altpleistozän von Hundsheim (Niederösterreich). Ann. Naturhist. Mus. Wien (Wien) 76:374-474.

RABEDER G., 1973a - Weitere Grabungebnisse von der alt-pleistozänen Wirbeltierfundstelle Deutsch-Altenburg 2. Die Höhle (Wien) 24:8-15.

RABEDER G., 1973b - Ein neuer Mustelide (Carnivora) aus dem Alt-Pleistozän von DeutschAltenburg 2 (Niederösterreich). N. Jb. Geol. Paläont. Mh. (Stuttgart) 1973(11):674-689.

RABEDER G., 1973c - Fossile Fledermausfaunen aus Österreich. Myotis (Bonn) 11:3-14.

RABEDER G., 1974a - Plecotus und Barbastella (Chiroptera) im Pleistozän von Österreich. Naturkundl. Jb. d. Stadt Linz (Linz) 1973: 159-184.

RABEDER G., 1974b - Fossile Schlangenreste aus den Höhlenfüllungen des Pfaffenberges bei Bad Deutsch-Altenburg (NÖ). Die Höhle (Wien) 25(4): 145-149.

RABEDER G., 1976a - Die Carnivoren (Mammalia) aus dem Altpleistozän von DeutschAltenburg 2. Beitr. Paläont. Österr (Wien) 1:5-119.

RABEDER G., 1978 - Das fossilführende Pleistozän-Profil im Höhlensystem «DeutschAltenburg 2-4-16») im Pfaffenberg bei Bad Deutsch-Altenburg (NÖ). Beitr. z. Quartär - und Landschaftsforschung, Festschrift für Julius FINK, (Kiel-Wien) im Druck. 